\title{
Análise da rede de multibormidades em idosos atendidos em um centro de referência: estudo transversal
}

\author{
Analysis of the multimorbidities \\ network in the elderly in a reference \\ center: a cross-section study
}

\author{
Manuella Franco Cerqueira da Silva ${ }^{1}$ \\ Cleber Luz-Santos ${ }^{2}$ (1) \\ Diana Oliveira Noronha ${ }^{3}$ (1) \\ Tainá Soares Risso Rattes ${ }^{4}$ (1)
}

\author{
Alanna Ribeiro da Silva ${ }^{5}$ (1) \\ Beatriz Santos Miranda ${ }^{6}$ (1) \\ Jessica Oliveira Barbosa 7 (i) \\ Kionna Oliveira Bernardes Santos ${ }^{8}$ (1)
}

'Autora para correspondência. Universidade Federal da Bahia (Salvador). Bahia, Brasil. manuella.franco@hotmail.com

2,4-8Universidade Federal da Bahia (Salvador).Bahia, Brasil.cleberluz@ufba.br, taina.rrattes@gmail.com, alannaribeiros@gmail.com, beamiranda13@

hotmail.com, jel_oliveira09@hotmail.com, kionna.ufba@gmail.com

${ }^{3}$ Centro de Referência Estadual de Atenção à Saúde do Idoso (Salvador). Bahia, Brasil. diananoronhafisio@gmail.com

RESUMO | INTRODUÇÃO: Idosos possuem múltiplos diagnósticos com padrões de similaridade que podem ser analisados pela teoria das redes complexas. Distúrbios crônicos com repercussões na capacidade funcional tendem aumentar em número e gravidade nesses indivíduos, sendo necessária análise integrada para compreender a relação entre envelhecimento e multimorbidades. OBJETIVO: Analisar os padrões de interação entre as doenças que acometem idosos de um centro de referência. MATERIAIS E MÉTODOS: Foram coletados dados clínicos e doenças diagnosticadas em 2973 prontuários de idosos cadastrados de um centro de referência de atenção à saúde do idoso. As doenças foram consideradas nós da rede e os idosos as arestas de modo não direcionada. O peso das arestas correspondiam à quantidade de idosos com duas doenças simultaneamente. Através do software Gephi (versão 0.9.2) foram analisados grau de conectividade entre as doenças (grau de entrada), interação das doenças em sugbrupos (Comunity), doenças mais influentes (Hubs) e doenças que atuam como pontes entre duas ou mais comunidades. RESULTADOS: Foram identificados 30 nós (doenças) e 433 arestas com pesos distintos de acordo com a quantidade de idosos, e 3 comunidades onde o grau de conectividade é maior entre si. A Hipertensão Arterial Sistêmica (HAS) apresentou-se como o Hub de maior influência na rede. CONCLUSÃO: A construção da rede complexa mapeou as doenças e a influência na assistência ao idoso. Informaç̃̃es relevantes para os gestores locais, na busca de intervenções efetivas no serviço que visem contribuir para prevenção das principais comorbidades e auxiliar na independência funcional dos pacientes.

PALAVRAS-CHAVE: Serviços de Saúde. Comorbidade. Idoso.
ABSTRACT | INTRODUCTION: Elderly people have multiple diagnoses with similar patterns that can be analyzed by the theory of complex networks. Chronic disorders with repercussions on functional capacity tend to increase in number and severity in these individuals, requiring an integrated analysis to understand the relationship between aging and multimorbidities. OBJECTIVE: To analyze patterns of interaction between diseases that affect elderly people in a reference center. MATERIALS AND METHODS: Clinical data and diagnosed diseases were collected from 2973 medical records of elderly people registered at a reference center for elderly health care. Diseases were considered nodes in the network and the elderly were considered to be rough edges. The weight of the edges corresponded to the number of elderly people with two diseases simultaneously. Through the Gephi software (version 0.9.2), the degree of connectivity between diseases (degree of entry), the interaction of diseases in subgroups (Community), most influential diseases (Hubs), and diseases that act as bridges between two or more communities were analyzed. RESULTS: 30 nodes (diseases) and 433 edges with different weights were identified according to the number of elderly people, and 3 communities where the degree of connectivity is greater among themselves. Systemic Arterial Hypertension (SAH) was the most influential hub in the network. CONCLUSION: The construction of the complex network mapped diseases and the influence on care for the elderly. Relevant information for local managers, in search of effective interventions in the service that aims to contribute to the prevention of the main comorbidities and assist in the functional independence of patients.

KEYWORDS: Health Services. Comorbidity. Aged. 


\section{Introdução}

As doenças que afetam uma população possuem padrões de similaridade que podem ser analisados pela teoria das redes complexas. Método de análise novo no contexto científico que avalia as interações entre as doenças em um determinado grupo de indivíduos ${ }^{1}$. Nos idosos as doenças crônicas tendem a aumentar em número e gravidade, sendo necessário extrapolar a análise isolada e compreendê-las em um contexto de rede.

Embora as evidências sejam incipientes nesta área, o estudo das relações entre as doenças já possui uma terminologia própria chamada de medicina em rede ${ }^{1}$. O foco da medicina em rede é identificar as conexões indiretas entre doenças aparentemente não conectadas ${ }^{1}$. Inicialmente desenvolvida para analisar as interações genômicas entre as doenças, seu uso tem sido ampliado para estudos epidemiológicos em comunidades ${ }^{2}$. Trata-se de um novo campo de pesquisa que surgiu na última década para abordar a complexidade biológica de modo integrado, potencializando a capacidade de decifrar a relação entre envelhecimento e multimorbidades 3 .

O processo de envelhecimento está associado à maior prevalência de doenças e distúrbios crônicos 4 com impacto direto na capacidade funcional, tempo de hospitalização e na mortalidade de pessoas idosas $^{5}$. Serviços de saúde para idosos vulneráveis que focam no tratamento de doenças isoladas terão dificuldades para entender problemas complexos de saúde e encontrar soluções efetivas para estes casos $^{6}$. Já que a maioria das doenças não transmissíveis está associada ao processo de envelhecimento e frequentemente coexistem no mesmo indivíduo ${ }^{7}$. A presença de duas ou mais doenças em um indivíduo com uma doença principal é definida como "comorbidade" e a presença de duas ou mais doenças no mesmo indivíduo sem referência a qualquer doença principal é definida como "multimorbidade"

O ponto de partida na análise das redes de doenças vai depender da perspectiva de quem a observa ${ }^{9}$. Existem três perspectivas: 1) Perspectiva genética - similaridades genéticas entre as doenças ${ }^{10}, 2$ ) Perspectiva metabólica - caminhos metabólicos compartilhados entre as doenças ${ }^{8}$ e a 3) Perspectiva da multimorbidade - coexistência de diferentes doenças no mesmo indivíduo além do esperado pelo acaso ${ }^{11}$.
As três podem ser utilizadas em conjunto ou separadas, mas a compreensão das interações das doenças entre os idosos num serviço de saúde já pode ser obtida sob a perspectiva da multimorbidade. Com isso, por meio do uso das redes complexas, esse estudo tem por objetivo analisar as características da rede de multimorbidades de idosos e secundariamente caracterizar o perfil desses indivíduos com cadastro ativo em um centro de referencia estadual de atenção à saúde do idoso.

\section{Metodologia}

\section{Escopo do estudo}

Foi realizado um estudo observacional do tipo transversal com dados secundários de prontuários dos idosos cadastrado no Centro de Referência Estadual de Atenção à Saúde do Idoso (CREASI). A coleta dos dados foi realizada entre fevereiro e outubro de 2019.

\section{Amostra e fonte dos dados}

Foram coletados os dados de todos os prontuários de idosos com cadastro ativo no Centro de Referência Estadual de Atenção à Saúde do Idoso (CREASI) até outubro de 2019. As informações foram obtidas por meio na Avaliação Multidimensional da Pessoa Idosa (AMPI) que é preenchida obrigatoriamente na admissão do paciente na unidade. A AMPI continha um checklist das doenças mais comuns nos idosos definidas pelo Assessing Care of Vulnerable Elders-3 (ACOVE $3)^{12}$. O formulário de coleta da pesquisa capturou as informações deste checklist e também dados sóciodemograficos para caracterização da amostra.

Este trabalho foi submetido e aprovado pelo Comitê de Ética em Pesquisa (CEP) em seres humanos da Secretária da Saúde do Estado da Bahia (SESAB) sob número 2.581.226 (CAAE 84753617.4.0000.0052). As recomendações propostas pela resolução 466/12 do Conselho Nacional de Saúde foram consideradas pelos pesquisadores durante sua execução.

\section{Checklist de doenças}

A AMPI possui uma lista com as morbidades mais comuns em idosos vulneráveis. Esta lista foi validada e padronizada pelo comitê gestor do Assessing Care 
of Vulnerable Elders-3 em $2006^{12}$ que contemplou 32 doenças na população idosa vulnerável ${ }^{13}$. O preenchimento do checklist foi realizado por médico geriatra durante a aplicação da AMPI. As doenças presentes no checklist foram: demência, doença de Parkinson, depressão, diabetes mellitus, hipertensão arterial sistêmica, doença arterial coronariana, insuficiência cardíaca (ICC), acidente vascular encefálico, fibrilação atrial, subnutrição, osteoartrite, osteoporose, pneumonia/influenza, úlcera por pressão, quedas e instabilidade postural, imobilidade, incontinência urinária e fecal, déficit visual, déficit auditivo, dor crônica, hipotireoidismo, continuidade do cuidado, manejo de medicamentos (polifarmácia), cuidado hospitalar e cuidado paliativo.

\section{Coleta de dados}

A coleta dos dados na AMPI foi realizada por 13 pesquisadores assistentes supervisionados pelos pesquisadores principais para minimizar coletas duplicadas e retrabalho por meio de revisões semanais da base de dados. Todos os pesquisadores receberam um treinamento prévio e realizaram a coleta por meio de um formulário eletrônico. A aplicação deste formulário foi realizada numa sala restrita do Núcleo de Ensino e Pesquisa (NEP) do CREASI. O local foi devidamente equipado para esta finalidade com até quatro computadores disponíveis para esta função. Em caso de dúvidas os pesquisadores puderam recorrer aos profissionais do NEP que conhecem com profundidade os itens da AMPI. Portanto, o banco foi tratado continuamente para garantir a confiabilidade das informações registradas. Após revisão final, em uma cópia, os dados de identificação foram substituídos por códigos alfanuméricos dos sujeitos, sendo liberada para elaboração da máscara de dados da rede de multimorbidades.

\section{Análise da rede de multimorbidades}

Os dados sociodemográficos dos idosos foram utilizados para caracterização da população do estudo. Foi realizada uma análise descritiva com medidas de tendência central para as variáveis quantitativas. Medidas de frequência foram utilizadas nas variáveis ordinárias e/ou dicotômicas para verificação das proporções. Esta análise foi realizada com auxílio do pacote estatístico Statistical Package for Social Sciences - Statistics for Windows (SPSS), versão 20.0
Os indivíduos foram categorizados, com base na classificação de fragilidade de Moraes et al., em três níveis, segundo seu estrato clínico-funcional: 1) Idoso Robusto (1 a 3), 2) Em Risco de Fragilização (4 e 5) e 3) Idoso Frágil (6 a 10) ${ }^{14}$.

A análise de rede foi realizada com auxílio do software Gephi (versão 0.9.2) e o layout adotado foi baseado do algoritmo ForceAtlas $2^{15}$. Todos os idosos com cadastro ativo no CREASI foram inseridos nesta rede, totalizando 2.973 idosos. Primeiramente foram definidos os nós que são as comorbidades. Em seguida foram estabelecidos os conjuntos de ligações a um mesmo nó o que estabelece o aparecimento de vértices centrais, os chamados hubs. Esses vértices que concentram mais ligações com outros têm maior probabilidade de criar novas ligações ${ }^{16}$.

O Grau de entrada (a quantidade de arestas que chegam ao nó) juntamente com a medida de centralidade (número total de caminhos mais curtos não redundantes que passam por um determinado nó) foram usados para avaliar a relevância da localização dos nós em uma rede ${ }^{9}$. Foram usadas três medidas bem conhecidas de centralidade, a saber, centralidade entre proximidade, centralidade de proximidade e centralidade de vetor próprio (disponível como parte do pacote Gephi). Em resumo, a centralidade do vetor próprio é uma medida da importância do nó em uma rede com base nas conexões de um nó, e a centralidade da proximidade é a distância média de um determinado nó inicial a todos os outros nós da rede. Definimos um cluster como uma parte de uma rede em que os nós são acessíveis apenas a partir de nós na mesma rede. Comunidade ou modularidade foram obtidos na rede com resolução de $0,6 \frac{17}{}$, esta medida representa maiores conexões entre os nós de um grupo específico em comparação com outros nós em toda a rede. $\mathrm{O}$ algoritmo de detecção de comunidades utilizado no Gephi é capaz de identificar os módulos em redes bipartidas como a deste estudo¹. Foi utilizado também o PageRank, que mede a importância de um vértice na rede considerando cada aresta. Quanto mais arestas recebidas por cada vértice adjacente, maior a importância ou autoridade desse vértice, o que indica a importância daquela comorbidade em um determinado grupo de pacientes. 


\section{Resultados}

Os resultados do processo de coleta estão descritos na Figura 1. Foram coletados 3.823 prontuários de idosos atendidos no CREASI. Foram excluídos 472 prontuários de pacientes inativos no serviço (87 altas, 103 abandonos e 282 óbitos). Além disso, foram excluídos 378 prontuários duplicados, restando 2.973 prontuários de pacientes com cadastro ativo, incluídos para a descrição deste estudo.

Figura 1. Fluxograma da seleção dos prontuários

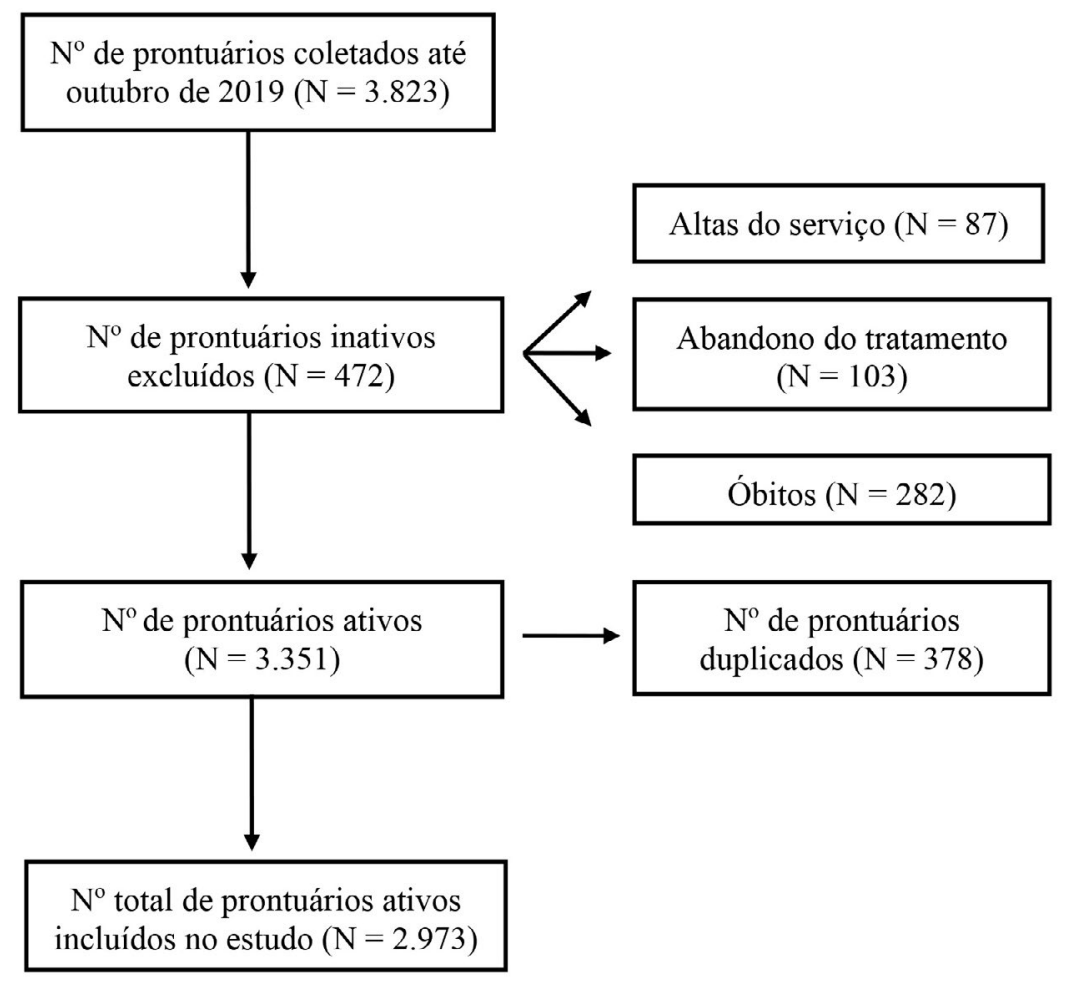

O maior percentual dos idosos possuíam idade variando entre 70 a 79 anos (38,9\%), eram majoritariamente do sexo feminino (71,9\%), com grau de escolaridade entre 1 a 7 anos de estudos $(48,9 \%)$, da cor/raça autodeclarada parda $(36,9 \%)$, casados $(20,8 \%)$, residentes na cidade Salvador $(90,4 \%)$. As características sociodemográficas desses indivíduos estão apresentadas na Tabela 1. 


\section{$\mathrm{N}$}

Idade $(n=2.973)$

60 a 69

70 a 79

80 a 89

90 a 99

100 ou mais

Sexo $(n=2.973)$

Feminino

Masculino

Escolaridade $(n=2.973)$

Analfabeto

8 anos ou mais

Dado não preenchido

Cor/Raça $(n=2.973)$

$\begin{array}{lcc}\text { Amarela } & 78 & 2,6 \\ \text { Branca } & 278 & 9,4 \\ \text { Indígena } & 7 & 0,2 \\ \text { Parda } & 1098 & 36,9 \\ \text { Preta } & 332 & 11,2 \\ \text { Ignorada } & 1180 & 39,7\end{array}$

\section{Estado Civil $(n=2.973)$}

Solteiro

Casado

Viúvo

Dado não preenchido

\section{Cidade $(n=2.973)$}

Salvador

Demais municípios da Bahia
2688

285
90,4

9,6

Fonte: Centro de Referência Estadual à Saúde do Idoso. 
Os estratos clínicos-funcionais mais predominantes foram o 1 com 17,6\%, 6 com o mesmo percentual de 17,6\% e 8 com 17,3\%. A maioria dos idosos $(61,9 \%)$ atendidos no serviço foi identificada como frágeis, em risco de fragilização representaram 19\% e robustos 19,2\% dos pacientes (Figura 2).

Figura 2. Estratificação clínico-funcional dos idosos atendidos no Centro de Referência Estadual a Atenção do Idoso em Salvador, Bahia

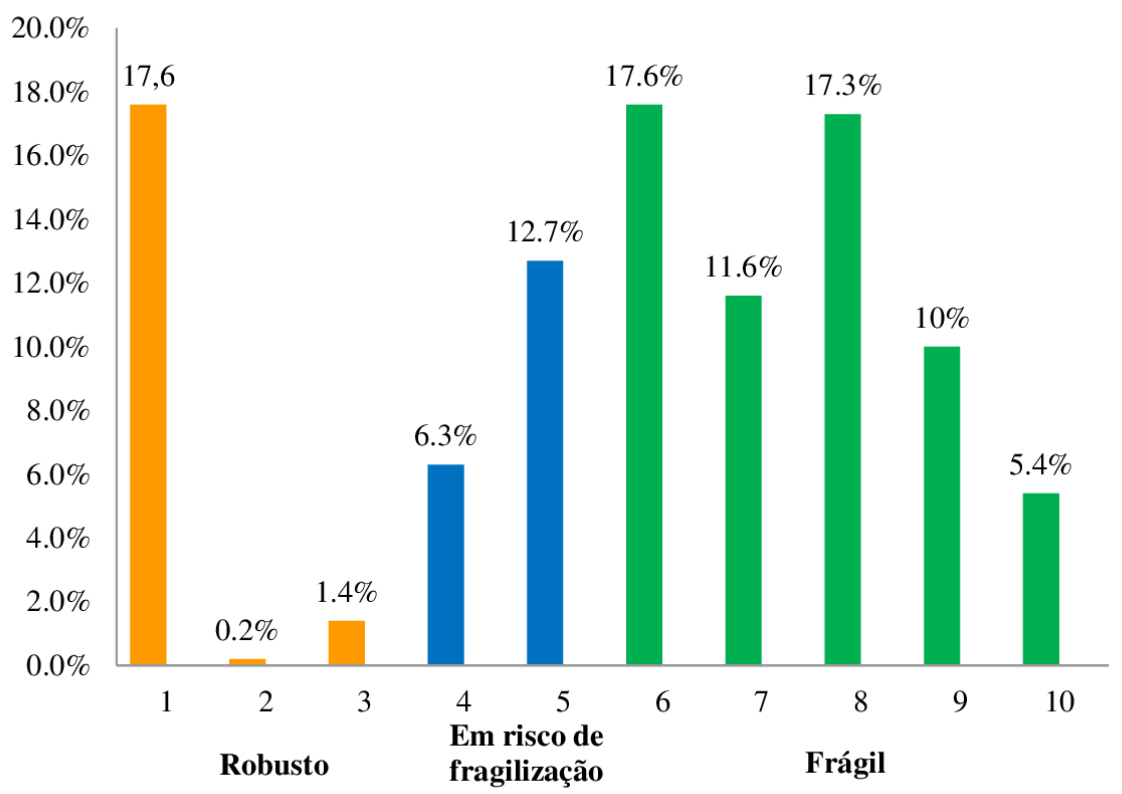

Ao todo, a rede bipartida compreendeu 30 nós com 443 arestas que são as ligações entre as doenças. As morbidades foram agrupadas em 3 comunidades interconectadas (nós que são acessíveis apenas a partir de nós na mesma rede), variando em tamanho e em cores (Figura 3). Quanto maior o tamanho do nó na rede, maior a sua importância, ou seja, mais conexões existem com aquela morbidade. E as cores foram selecionadas de acordo com as morbidades que estão na mesma comunidade.

Figura 3. Representação da rede de doenças segundo as comunidades interconectadas

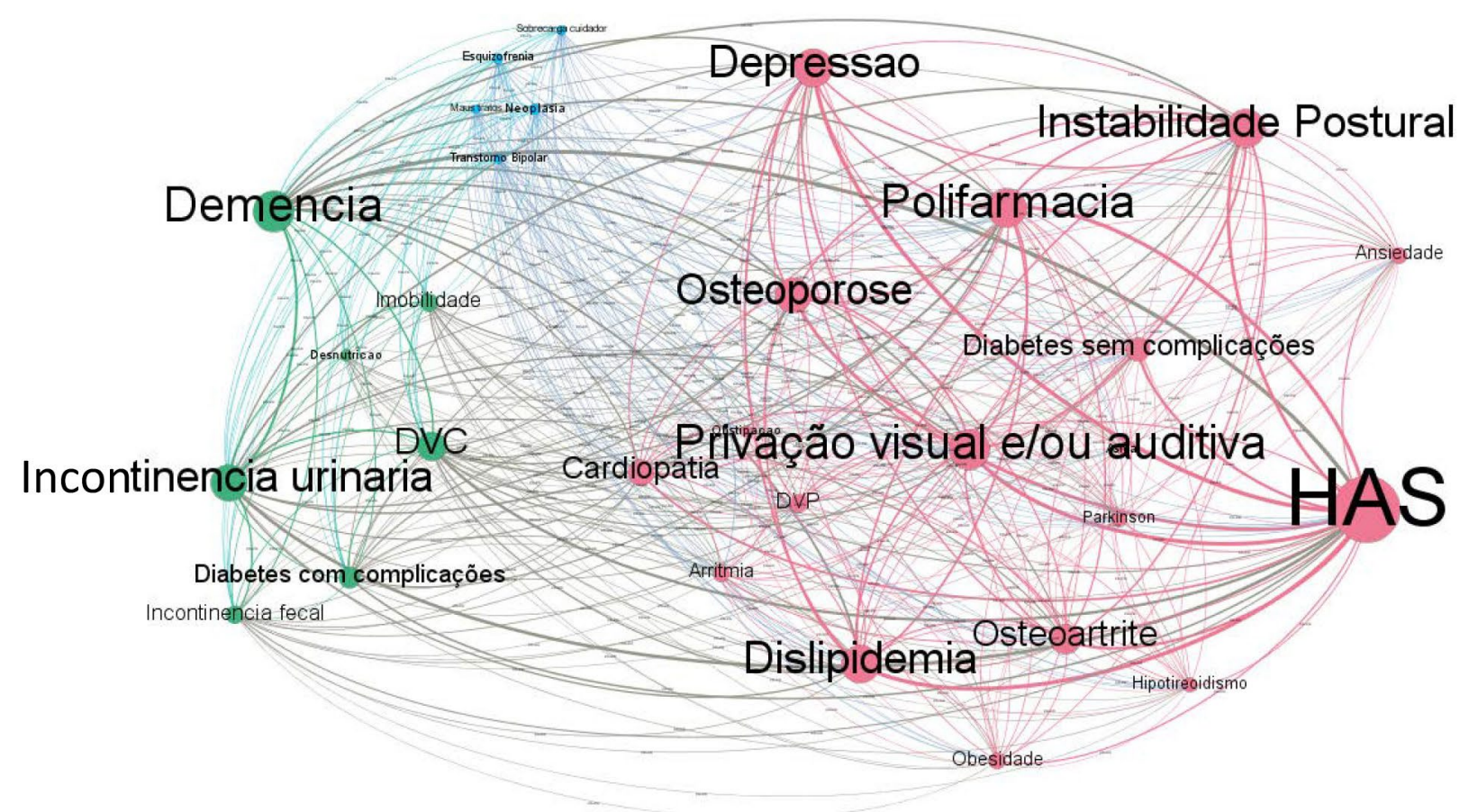


A construção da rede também permitiu detectar as doenças com maior relevância, as que apresentam mais conexões com maior grau de entrada, como a HAS que é a doença que possui maior relevância com 2.084 conexões, seguida da depressão com 1.063, demência com 1.043, dislipidemia com 932 e osteoporose com 889, como demonstrado na Tabela 2. Ainda, a identificação dos idosos com maior predisposição ao surgimento de novas morbidades, foi possível de ser analisada através da medida de força de conectividade, de acordo com a análise das variáveis PageRrank e Grau de Entrada (Tabela 2).

O PageRrank também permite a avaliação da presença de influenciadores, ou seja, nós que possuem extrema relevância ou autoridade. As cinco doenças com maior índice de autoridade foram ordenadas da maior para menor entre elas HAS, demência, depressão, dislipidemia e osteoporose respectivamente (Tabela 2).

Tabela 2. Apresentação das variáveis de PageRrank e Grau de Entrada

\begin{tabular}{ll}
\hline PageRank & Grau de entrada \\
\hline HAS 0,080077 & HAS 2084 \\
Demência 0,0405 & Depressão 1063 \\
Depressão 0,040417 & Demência 1043 \\
Dislipidemia 0,030313 & Dislipidemia 932 \\
Osteoporose 0,029795 & Osteoporose 889 \\
\hline
\end{tabular}

Fonte: Centro de Referência Estadual à Saúde do Idoso.

\section{Discussão}

O envelhecimento torna o indivíduo mais suscetível e vulnerável a múltiplas doenças crônicas, incapacidades funcionais, o que exige atendimentos multidisciplinares, internações hospitalares, maiores gastos e desafia o sistema de saúde ${ }^{18}$. Por meio da construção de uma rede complexa é possível entender o comportamento e a interação dessas doenças em uma determinada população.

O uso de redes para modelar sistemas é uma informação pouco utilizada na área da saúde, no entanto, ela permite representar e compreender problemas como a identificação da doença mais comum em um grupo específico, assim como mapeia as doenças que estão mais associadas a aqueles indivíduos.

Uma rede corresponde a um grafo, que é representado por um conjunto de vértices (nós) ligados por arestas (linhas), que estabelecem uma relação entre si, ou seja, cada linha tem uma direção que conecta um nó a outro nó ${ }^{19}$. Nessa rede os pacientes e as comorbidades representam os nós e o sentindo das ligações ou arestas sempre partem do paciente para a doença, nunca há ligações inter grupos.

Os dados descrevem que os idosos atendidos no CREASI são caracterizados como mulheres, entre 70 a 79 anos, casadas, pardas, com nível de escolaridade de 1 a 7 anos e residentes na capital baiana, Salvador. Apesar de ser um centro de referência estadual, o serviço da atenção secundária atende apenas $9,6 \%$ de idosos provenientes de cidades do interior, e é o único centro de referência para a saúde da população idosa no estado.

O envelhecimento está associado ao processo de fragilização multidimensional em que ocorre redução da capacidade de adaptação às agressões biopsicossociais e, consequentemente, aumenta a vulnerabilidade ao declínio funcional e suas repercussões. Através da aplicação da Avaliação Multidimensional da Pessoa Idosa (AMPI) é possível classificar os idosos em dez categorias clínico-funcionais 20 , que é baseada na funcionalidade (dependência ou independência para atividades), na presença de fatores de risco, doenças e comorbidades ${ }^{20}$. Na análise dos dados, foi identificada a presença da maior parte de idosos classificados como frágeis (61,9\%), ou seja, que apresentam um declínio funcional e necessitam de maior auxilio para realizar as atividades de vida diária ${ }^{14}$. 
Os estratos mais prevalentes nos idosos foram o 6 e 8 , que indicam que os idosos apresentam declínio funcional parcial nas atividades instrumentais de vida diária, mas que permanecem independentes nas atividades básicas da vida diária (ABVD); e idosos que apresentam declínio funcional total nas atividades instrumentais da vida diária (AIVD), bem como semidependência nas $A B V D$, respectivamente ${ }^{14}$. A partir dessa estratificação é possível identificar e monitorar os grupos e direcionar um planejamento e tratamento adequado para cada um, sobretudo ao idoso frágil que possui alto grau de dependência e necessita de maior atenção ${ }^{20}$.

A hipertensão arterial sistêmicafoi à condição de saúde que teve mais conexões e maior relevância na rede, sendo umas das cinco doenças mais presentes nesses indivíduos (2.084). Só no Brasil 60,9\% das pessoas com mais de 65 anos apresentam essa doença, que é um dos principais fatores de risco para ocorrência de doenças cardiovasculares e cerebrais ${ }^{21}$. No ano de 2017, o país registrou 141.878 óbitos devido à hipertensão ou a causas atribuíveis a ela. Apesar do alto risco de mortalidade, é uma doença que muitas vezes pode ser evitada com hábitos de vida mais saudáveis e prática regular de exercício físico ${ }^{22}$, sendo possível seu tratamento no nível de atenção primária à saúde.

A presença de condições de saúde crônicas aumenta a complexidade do manejo clínico, implicando no aumento do risco de iatrogenia. O declínio funcional e a fragilidade estão intimamente relacionados ${ }^{14}$. Além disso, a ocorrência de uma morbidade geralmente leva a existência de outras sucessivamente. Por ser uma doença de base muito comum na população brasileira, a hipertensão normalmente influência na existência de outras, por exemplo, indivíduos hipertensos fazem uso de diuréticos poupadores de potássio, que aumentam o risco de distúrbios hidro-eletrolíticos, que é uma das causas de arritmia ${ }^{23}$.

Essa análise é de fundamental importância para os gestores do local, que podem obter informações da atual situação dos pacientes e com isso, buscar intervenções efetivas através de serviços e ações que visem contribuir para prevenção das principais comorbidades apresentadas por esses indivíduos, buscando o envelhecimento ativo e saudável, tendo em vista a independência funcional e autonomia dos idosos.
Esse estudo apresenta algumas limitações. Inicialmente, por se tratar de estudo de natureza observacional do tipo transversal baseado na análise de prontuários, o que aumenta o risco de vieses no resultado final, uma vez que não é o mesmo avaliador que preenche todos os prontuários. Além disso, como vários pesquisadores coletaram e preencheram o banco de dados de forma manual, algum erro pode ter passado despercebido, mesmo com a revisão dos pesquisadores principais.

\section{Conclusão}

O mapeamento da rede de doenças demonstrou a influência das comorbidades na rede de assistência ao idoso frágil do CREASI, tendo como principais influentes a HAS, depressão, demência, dislipidemia, osteoporose e privação visual e/ou auditiva. Além disso, a análise permitiu avaliar o perfil dos idosos atendidos, caracterizado por indivíduos frágeis, do sexo feminino e que possuem como principal doença a HAS, além de duas ou mais comorbidades.

\section{Agradecimentos}

Ao Centro de Referência Estadual de Atenção à Saúde do Idoso, pelos dados cedidos para esta pesquisa. Á Universidade Federal da Bahia (UFBA) pela concessão de bolsa de iniciação cientifica a Manuella Franco Cerqueira da Silva, pelo PIBIC. E ao professor José Garcia Vivas Miranda pelas suas contribuições na análise, modelamento e construção da rede de comorbidades.

\section{Contribuições dos autores}

Silva MFC, Santos CL participaram da concepção, delineamento, coleta de dados, busca e análise estatística dos dados da pesquisa, interpretação dos resultados e redação do artigo científico. Santos DOL participou da concepção, delineamento, coleta de dados, busca e análise estatística dos dados da pesquisa. Rattes TSR, Silva AR, Miranda BS, Barbosa JO participaram da concepção, coleta de dados, busca e análise estatística, interpretação dos dados e contribuições relevantes para escrita do artigo científico. Bernardes KO participou da concepção, delineamento, interpretação dos resultados, escrita e revisão do artigo científico.

\section{Conflitos de interesses}

Nenhum conflito financeiro, legal ou político envolvendo terceiros (governo, empresas e fundações privadas, etc.) foi declarado para nenhum aspecto do trabalho submetido (incluindo, mas não se limitando a subvenções e financiamentos, participação 
em conselho consultivo, desenho de estudo, preparação de manuscrito, análise estatística, etc.).

\section{Referências}

1. Carson MB, Lu H. Network-based prediction and knowledge mining of disease genes. BMC MedGenomics. 2015;(Suppl 2):9. doi: $10.1186 / 1755-8794-8-S 2-S 9$

2. Barabási AL, Gulbahce N, Loscalzo J. Network medicine: A network-based approach to human disease. Nat Rev Genet. 2011;12(1):56-68. doi: 10.1038/nrg2918

3. Faner R, Cruz T, López-Giraldo A, Agustí A. Network medicine, multimorbidity and the lung in the elderly. Eur Respir J. 2014;44(3):775-88. doi: 10.1183/09031936.00078714

4. Bousquet J, Anto JM, Sterk PJ, Adcock IM, Chung KF, Roca J et al. Systems medicine and integrated care to combat chronic noncommunicable diseases. Genome Med. 2011;3(7):43. doi: $10.1186 / g m 259$

5. Held FP, Blyth F, Gnjidic D, Hirani V, Naganathan V, Waite LM et al. Association Rules Analysis of Comorbidity and Multimorbidity: The Concord Health and Aging in Men Project. J Gerontol A Biol Sci Med Sci. 2016;71(5):625-31. doi: 10.1093/gerona/glv181

6. Banerjee S. Multimorbidity - Older adults need health care that can count past one. Lancet. 2015;385(9968):587-589. doi: $10.1016 /$ s0140-6736(14)61596-8

7. Anderson G. Chronic Care: Making the Case for Ongoing Care. Princeton, NJ: Robert Wood Johnson Foundation [Internet]. 2010 [accessed in 2020 mar 22]. Available at: https://www.rwjf.org/en/ library/research/2010/01/chronic-care.html

8. Van Den Akker M, Buntinx F, Knottnerus JA. Comorbidity or multimorbidity: What's in a name? A review of literature. Eur J Gen Pract. 1996;2(2):65-70. doi: 10.3109/13814789609162146

9. Goh K I, Cusick ME, Valle D, Childs B, Vidal M, Barabási AL. The human disease network. Proc Natl Acad Sci U S A. 2007;104(21):8685-90. doi: 10.1073/pnas.0701361104

10. Menche J, Sharma A, Kitsak M, Ghiassian SD, Vidal M, Loscalzo J et al. Disease networks. Uncovering disease-disease relationships through the incomplete interactome. Science. 2015;347(6224):1257601. doi: 10.1126/science.1257601

11. Girvan M, Newman MEJ. Community structure in social and biological networks. PNAS. 2002;99(12):7821-6. doi: 10.1073/ pnas. 122653799

12. Wenger NS, Roth CP, Shekelle P. Introduction to the Assessing Care of Vulnerable Elders-3 quality indicator measurement set. J Am Geriatr Soc. 2007;(Suppl 2):247-52. doi: 10.1111/j.15325415.2007.01328.x
13. MacLean CH, Louie R, Shekelle PG, Roth CP, Saliba D, Higashi $T$ et al. Comparison of administrative data and medical records to measure the quality of medical care provided to vulnerable older patients. Med Care. 2006;44(2):141-8. doi: 10.1097/01. mlr.0000196960.12860.de

14. Moraes EN, Lanna FM, Santos RR, Bicalho MAC, Machado C), Romero DE. A new proposal for the clinical-functional categorization of the elderly: Visual Scale of Frailty (VS-Frailty). J Aging Res Clin Pract. 2016;5(1):24-30. doi: 10.14283/jarcp.2016.84

15. Jacomy M, Venturini T, Heymann S, Bastian M. ForceAtlas2, a continuous graph layout algorithm for handy network visualization designed for the Gephi software. PLoSOne. 2014;9(6):e98679. doi: 10.1371/journal.pone.0098679

16. Diederichs C, Berger K, BartelsDB. The Measurement of Multiple Chronic Diseases-A Systematic Review on Existing Multimorbidity Indices. J Gerontol A Biol Sci Med Sci. 2011;66(3):301-11. doi: 10.1093/gerona/glq208

17. Hidalgo CA, Blumm N, Barabási AL, Christakis NA. A Dynamic Network Approach for the Study of Human Phenotypes. PLoS Comput Biol. 2009;5(4):e1000353. doi: 10.1371/journal. pcbi. 1000353

18. Miranda GMD, Mendes ACG, Silva ALA. O envelhecimento populacional brasileiro: desafios e consequências sociais atuais e futuras. Rev Bras Geriatr Gerontol. 2016;19(3):507-519. doi: 10.1590/1809-98232016019.150140

19. Metz J, Calvo R, Seno ERM, Romero RAF, Liang Z. Redes Complexas: conceitos e aplicações. Relatório Técnico. Instituto de Ciências Matemáticas e de Computação, São Carlos [Internet]. 2007. Disponível em: http://repositorio.icmc.usp. br/bitstream/handle/RIICMC/6720/Relat\%c3\%b3rio\%20 T\%c3\%a9cnico_290_2007.pdf?sequence=3

20. Moraes EN, Carmo JA, Moraes FL, Azevedo RS, Machado CJ, Montilla DER. Índice de Vulnerabilidade Clínico Funcional-20 (IVCF20): reconhecimento rápido do idoso frágil. Rev Saúde Pública. 2016;50:81. doi: 10.1590/s1518-8787.2016050006963

21. Ministério da Saúde. Hipertensão é diagnosticada em $24,7 \%$ da população, segundo a pesquisa Vigitel [Internet]. 2019. Disponível em: https://bvsalud.org/portal-lis/lis-search/ resource/?id=46541

22. Ministério da Saúde. Secretaria de Atenção Primária à Saúde. Sociedade Beneficente Israelita Brasileira Albert Einstein. Nota técnica para organização da rede de atenção à saúde com foco na atenção primária à saúde e na atenção ambulatorial especializada - saúde da pessoa idosa. São Paulo: Hospital Israelita Albert Einstein, 2019. 56 p.: il.

23. Dutra VF, Tallo FS, Rodrigues FT, Vendrame LS, Lopes RD, Lopes AC. Desequilíbrios hidroeletrolíticos na sala de emergência. Rev Soc Bras Clin Med. 2012;10(5):410-9. 